\title{
A CYCLIC INEQUALITY AND AN EXTENSION OF IT. II.
}

\author{
by P. H. DIANANDA
}

(Received 3rd April 1962)

\section{Introduction}

Throughout this paper, unless otherwise stated, $n$ and $L$ stand for positive integers and $\alpha, t, x, x_{1}, x_{2}, \ldots$ for positive real numbers. Let

where

$$
S_{n}\left(x_{1}, \ldots, x_{n}\right)=\sum_{r=1}^{n} \frac{x_{r}}{x_{r+1}+x_{r+2}},
$$

and

$$
x_{n+r}=x_{r}(\text { all } r)
$$

$$
\lambda(n)=\frac{1}{n} \inf _{x_{1}, \ldots, x_{n}} S_{n}\left(x_{1}, \ldots, x_{n}\right)
$$

Then, it is known (see (2)) that

$$
\begin{aligned}
\lambda(n)=\frac{1}{2} & (n \leqq 8), \\
& \left.<\frac{1}{2} \quad \text { (even } n \geqq 14, \text { odd } n \geqq 27\right) .
\end{aligned}
$$

Also, as Rankin (4) has proved, $\lambda(n)$ has a finite limit as $n \rightarrow \infty$ and

$$
\lambda=\lim _{n \rightarrow \infty} \lambda(n)=\inf _{n} \lambda(n) .
$$

Further (6),

$$
\lambda \leqq \lambda(24)<0 \cdot 49950317 \text {. }
$$

In a paper (1) to appear shortly, we have shown that

$$
\lambda(n) \geqq \lambda \geqq \frac{1}{2}\left(\sqrt{ } 2-\frac{1}{2}\right)=0.457107,
$$

thus improving Rankin's result (5)

$$
\lambda(n) \geqq \lambda \geqq 0 \cdot 330232,
$$

which he obtained by a method involving the use of properties of convex functions. Our result (6) was first obtained by a development of Rankin's method, although later a simpler proof was found (see (1)). In this paper we shall develop Rankin's method further and prove that

$$
\lambda(n) \geqq \lambda \geqq 0 \cdot 461238 \text {. }
$$

We shall also prove that

$$
\lambda \leqq \lambda(24)<0 \cdot 499197 .
$$


The upper and lower bounds for $\lambda$, appearing in (7) and (8), have a gap which is less than 90 per cent. of that between the best previously known bounds which are given in (5) and (6).

\section{Some Lemmas}

Lemma 1. Let $\alpha, x_{1}, x_{2}, \ldots$ be any real numbers satisfying (2). Then there are integers $a_{1}, a_{2}, \ldots, a_{s+1}(s>0)$, with

$$
a_{s+1} \equiv a_{1}(\bmod n)
$$

such that, for $k=1,2, \ldots, s$,

$$
a_{k+1} \geqq a_{k}+2
$$

and

(ii) either $\quad a_{k+1}-a_{k}$ is even, $x_{a_{k+1}} \geqq \alpha x_{a_{k+1}+1}$

$$
\left.\begin{array}{ll}
\text { and } & x_{k+1}-a_{k} \text { is even, } x_{a_{k+1}} \geqq \alpha x_{a_{k+1}+1} \\
\text { or } & x_{a_{k}+2}<\alpha x_{a_{k}+3}<x_{a_{k}+4}<\alpha x_{a_{k}+5}<\ldots<x_{a_{k+1}},
\end{array}\right\}
$$

Proof. First let $a_{k}$ be an arbitrary integer. Consider the infinite chain $C$ of inequalities

$$
x_{a_{k}+2}<\alpha x_{a_{k}+3}<x_{a_{k}+4}<\alpha x_{a_{k}+5}<x_{a_{k}+6}<\ldots .
$$

If all these inequalities are true then

and so

$$
x_{a_{k}+2}<x_{a_{k}+4}<x_{a_{k}+6}<\ldots
$$

$$
x_{a_{k}+2}<x_{a_{k}+2 n+2} .
$$

This contradicts (2). Hence the inequalities in $C$ are not all true. Suppose that the first $b_{k}=a_{k+1}-\left(a_{k}+2\right)$ inequalities in $C$ are true and the next one false. Then we have (10) since $b_{k} \geqq 0$. Also we have (11) if $b_{k}$ is even and (12) if $b_{k}$ is odd. Thus there is an integer $a_{k+1}$ satisfying both (i) and (ii).

Hence, starting with an arbitrary integer $a_{1}$, we can find successively integers $a_{2}, a_{3}, a_{4}, \ldots$ satisfying (i) and (ii) for $k=1,2,3, \ldots$ respectively. Consider now the infinite sequence of integers $a_{1}, a_{2}, a_{3}, \ldots$. Since there are only $n$ residue classes $(\bmod n)$, it follows that there are positive integers $s$ and $t$ such that $a_{s+t} \equiv a_{t}(\bmod n)$. Also (i) and (ii) are satisfied for $k=t$, $t+1, \ldots, s+t-1$. Since (for any fixed $s$ and $t$ ) $a_{t}, a_{t+1}, \ldots, a_{s+}$ can be renamed $a_{1}, a_{2}, \ldots, a_{s+1}$ respectively, the lemma follows. (I am indebted to the referee for commenting that my original proof needed further clarification.)

Following Rankin (5), we write

$$
\left(\phi_{L} x_{0}, x_{1}, \ldots, x_{L+1}\right)=\sum_{r=0}^{L-1} \frac{x_{r}}{x_{r+1}+x_{r+2}} .
$$


We write also

$$
\begin{aligned}
& \psi_{L}\left(x_{0}, x_{1}, \ldots, x_{L}\right)=\frac{x_{0}}{x_{1}+x_{2}}+\frac{x_{1}+x_{2}}{x_{3}+x_{4}}+\frac{x_{3}+x_{4}}{x_{5}+x_{6}}+\ldots+\frac{x_{L-3}+x_{L-2}}{x_{L-1}+x_{L}} \\
& \left.+\frac{\alpha x_{L-1}}{(1+\alpha) x_{L}} \text { (even } L \geqq 2\right) \text {, } \\
& =\frac{x_{0}}{x_{1}+x_{2}}+\frac{x_{1}+x_{2}}{x_{3}+x_{4}}+\frac{x_{3}+x_{4}}{x_{5}+x_{6}}+\ldots+\frac{x_{L-4}+x_{L-3}}{x_{L-2}+x_{L-1}} \\
& \left.+\frac{x_{L-2}+x_{L-1}}{(1+\alpha) x_{L}} \text { (odd } L \geqq 3\right) \text {. }
\end{aligned}
$$

Lemma 2. Let $L$ be even and $\geqq 2$. Suppose that

$$
x_{2}<\alpha x_{3}<x_{4}<\alpha x_{5}<\ldots<x_{L} \text { and } x_{L} \geqq \alpha x_{L+1} .
$$

Then

$$
\phi_{L}\left(x_{0}, x_{1}, \ldots, x_{L+1}\right) \geqq \psi_{L}\left(x_{0}, x_{1}, \ldots, x_{L}\right) .
$$

Proof. This follows from (13), (14) and (16).

Lemma 3. Let $L$ be odd and $\geqq 3$. Suppose that

$$
x_{2}<\alpha x_{3}<x_{4}<\alpha x_{5}<\ldots<\alpha x_{L} \text { and } \alpha x_{L} \geqq x_{L+1} .
$$

Then (17) is true.

Proof. This follows from (13), (15) and (18).

For each $t$, we define functions $f_{t}(x), g_{t}(x), F_{t}(x)$ and $G_{t}(x)$ for $x \geqq 0$ as follows.

$$
\begin{aligned}
& f_{t}(x)=\frac{1}{2} t x^{2 / t} \\
& =\frac{1}{2}(t+2)\left(\frac{\alpha x}{1+\alpha}\right)^{2 /(t+2)}-\frac{\alpha}{1+\alpha} \text { for } x \geqq\left(\frac{\alpha}{1+\alpha}\right)^{\frac{1}{2} L} . \\
& g_{t}(x)=\frac{1}{2}(t+1)\left(\frac{x}{1+\alpha}\right)^{2 /(t+1)} \text {. } \\
& F_{t}(x)=\frac{2}{t} f_{t}\left(x^{\frac{1 t}{2}}\right)=x \quad \text { for } x \leqq \frac{\alpha}{1+\alpha}, \\
& =\frac{t+2}{t}\left(\frac{\alpha}{1+\alpha}\right)^{2 /(t+2)} x^{t /(t+2)}-\frac{2}{t} \frac{\alpha}{1+\alpha} \text { for } x \geqq \frac{\alpha}{1+\alpha} \text {. } \\
& G_{t}(x)=\frac{2}{t} g_{t}\left(x^{\frac{1}{t}}\right)=\frac{t+1}{t}\left(\frac{1}{1+\alpha}\right)^{2 /(t+1)} x^{t /(t+1)} .
\end{aligned}
$$

The functions defined above are all convex functions of $\log x$ for $x>0$, but we shall use this convexity property only of $F_{2}(x)$ and $G_{3}(x)$. 


\section{Lemma 4.}

and

$$
\left.\begin{array}{rl}
F_{2}(x) & =x \text { for } x \leqq \frac{\alpha}{1+\alpha}, \\
& =2 \sqrt{\frac{\alpha x}{1+\alpha}}-\frac{\alpha}{1+\alpha} \text { for } x \geqq \frac{\alpha}{1+\alpha} ;
\end{array}\right\}
$$

are convex functions of $\log x$ for $x>0$. Also

$$
F_{2}(x) \geqq 2 \sqrt{\frac{\alpha x}{1+\alpha}}-\frac{\alpha}{1+\alpha} .
$$

Proof. The convexity properties follow from the following facts: For $x>0$ (i) $F_{2}(x)$ and $G_{3}(x)$ are continuous and have continuous derivatives, (ii) except at $x=\frac{\alpha}{1+\alpha}, F_{2}^{\prime \prime}(x)$ exists and $x F_{2}^{\prime \prime}(x)+F_{2}^{\prime}(x) \geqq 0$ and (iii) $G_{3}^{\prime \prime}(x)$ exists and $x G_{3}^{\prime \prime}(x)+G_{3}^{\prime}(x) \geqq 0$.

(25) follows from (23) since $x+\frac{\alpha}{1+\alpha} \geqq 2 \sqrt{\frac{\alpha x}{1+\alpha}}$, by the inequality of the (arithmetic and geometric) means.

Lemma 5. $F_{t}(x) \geqq F_{t^{\prime}}(x)$ for $t \geqq t^{\prime}>0$.

Proof. From (21), the result is true for $x \leqq \frac{\alpha}{1+\alpha}$. When $x \geqq \frac{\alpha}{1+\alpha}$,

$$
F_{t}^{\prime}(x)-F_{t^{\prime}}^{\prime}(x)=\left(\frac{\alpha}{(1+\alpha) x}\right)^{2 /(t+2)}-\left(\frac{\alpha}{(1+\alpha) x}\right)^{2 /\left(t^{\prime}+2\right)} \geqq 0
$$

Hence

$$
F_{t}(x)-F_{t^{\prime}}(x) \geqq F_{t}\left(\frac{\alpha}{1+\alpha}\right)-F_{t^{\prime}}\left(\frac{\alpha}{1+\alpha}\right)=0 .
$$

Lemma 6. $G_{1}(x) \geqq F_{2}(x)$ if

$$
t>1 \text { and } \alpha(1+\alpha) \leqq\left(\frac{t}{t-1}\right)^{t-1}
$$

Proof. Let

$$
F(x)=2 \sqrt{\frac{\alpha x}{1+\alpha}}-\frac{\alpha}{1+\alpha} .
$$

Then, from (22) and (27),

$$
\begin{aligned}
G_{t}^{\prime}(x)-F^{\prime}(x) & =(1+\alpha)^{-2 /(t+1)} x^{-1 /(t+1)}-\left(\frac{\alpha}{1+\alpha}\right)^{\frac{1}{2}} x^{-\frac{1}{2}} \\
& =0 \text { at } x=\frac{\alpha}{1+\alpha}\{\alpha(1+\alpha)\}^{2 /(t-1)},
\end{aligned}
$$


where $G_{t}(x)-F(x)$ has the minimum value

$$
\frac{\alpha}{1+\alpha}-\frac{t-1}{t} \alpha^{t /(t-1)}(1+\alpha)^{(2-t) /(t-1)} \geqq 0,
$$

from (26). Hence $G_{t}(x) \geqq F(x)=F_{2}(x)$ for $x \geqq \frac{\alpha}{1+\alpha}$, from (23) and (27).

For $x \leqq \frac{\alpha}{1+\alpha}, G_{t}(x) \geqq F_{2}(x)$ is equivalent, from (22) and (27), to

$$
x \leqq\left(\frac{t+1}{t}\right)^{t+1}(1+\alpha)^{-2}
$$

which is satisfied if $\alpha(1+\alpha) \leqq\left(\frac{t+1}{t}\right)^{t+1}$. But (26) is true, and

$$
\left(\frac{t}{t-1}\right)^{t-1} \leqq\left(\frac{t+1}{t}\right)^{t+1} \text { since }\left(\frac{t}{t-1}\right)^{t-1}\left(\frac{t}{t+1}\right)^{t+1} \leqq 1
$$

by the inequality of the means. Hence $G_{t}(x) \geqq F_{2}(x)$ for $x \leqq \frac{\alpha}{1+\alpha}$ also.

Lemma 7. $\psi_{\mathbf{L}}\left(x_{0}, x_{1}, \ldots, x_{L}\right) \geqq f_{L}\left(x_{0} / x_{L}\right) \quad$ (even $\left.L \geqq 2\right)$,

$$
\geqq g_{L}\left(x_{0} / x_{L}\right) \quad(\text { odd } L \geqq 3) \text {. }
$$

Proof. For odd $L \geqq 3$, (29) follows from (15), (20) and the inequality of the means. Let $x=x_{0} / x_{L}$.

For even $L \geqq 2$ and $x \geqq\left(\frac{\alpha}{1+\alpha}\right)^{\frac{1}{2} L}$,

$$
\begin{aligned}
\psi_{L}\left(x_{0}, x_{1}, \ldots, x_{L}\right)+\frac{\alpha}{1+\alpha}=\frac{x_{0}}{x_{1}+x_{2}}+\frac{x_{1}+x_{2}}{x_{3}+x_{4}} & +\frac{x_{3}+x_{4}}{x_{5}+x_{6}}+\ldots \\
& +\frac{x_{L-3}+x_{L-2}}{x_{L-1}+x_{L}}+\frac{\alpha\left(x_{L-1}+x_{L}\right)}{(1+\alpha) x_{L}},
\end{aligned}
$$

from (14). Hence (28) follows from the inequality of the means and (19).

For even $L \geqq 2$ and $x \leqq\left(\frac{\alpha}{1+\alpha}\right)^{\frac{1}{2} L}$,

$$
\psi_{L}\left(x_{0}, x_{1}, \ldots, x_{L}\right) \geqq \frac{1}{2} L\left(\frac{x_{0}}{x_{L-1}+x_{L}}\right)^{2 / L}+\frac{\alpha}{1+\alpha} \frac{x_{L-1}}{x_{L}},
$$

from (14) and the inequality of the means. Hence

where

$$
\psi_{L}\left(x_{0}, x_{1}, \ldots, x_{L}\right) \geqq h\left(\frac{x_{0}}{x_{L}}, \frac{x_{L-1}}{x_{L}}\right)
$$

$$
h(x, u)=\frac{1}{2} L\left(\frac{x}{1+u}\right)^{2 / L}+\frac{\alpha u}{1+\alpha}
$$


Now

$$
\begin{aligned}
\frac{\partial}{\partial u} h(x, u)=-\frac{1}{1+u}\left(\frac{x}{1+u}\right)^{2 / L}+\frac{\alpha}{1+\alpha} & \\
& \geqq 0 \text { for } u \geqq 0 \text { and } x \leqq\left(\frac{\alpha}{1+\alpha}\right)^{\frac{1}{2 L}} \text {. }
\end{aligned}
$$

Thus $\psi_{L}\left(x_{0}, x_{1}, \ldots, x_{L}\right) \geqq h\left(x_{0} / x_{L}, 0\right)=f_{L}\left(x_{0} / x_{L}\right)$, from (19). This completes the proof of the lemma.

Lemma 8. $\left(\frac{t}{t-1}\right)^{t-1}$ increases for $t>1$.

Proof. This follows from Theorem 140 of (3).

Lemma 9. $G_{t}(x) \geqq F_{2}(x)$ for $t \geqq 3$ if $\alpha(1+\alpha) \leqq \frac{9}{4}$.

Lemma 10. $G_{t}(x) \geqq F_{2}(x)$ for $t \geqq 5$ if $\alpha(1+\alpha) \leqq \frac{625}{256}$.

Proofs. Lemmas 9 and 10 follow from Lemmas 6 and 8 .

Lemma 11. $\sqrt{ } x-\frac{1}{2} x$ increases for $0 \leqq x \leqq 1$.

Lemma 12. $(1+x) e^{-x}$ decreases for $x \geqq 0$.

Proofs. Lemmas 11 and 12 have obvious proofs.

\section{First Improvement of (6)}

We can improve (6) to

$$
\lambda(n) \geqq \lambda \geqq \frac{8 \sqrt{10}-17}{18}=0.461012,
$$

without much computational work, as follows.

Let $\alpha, x_{1}, x_{2}, \ldots$ be positive and (2) be satisfied. Then we can find an increasing sequence of integers $a_{1}, a_{2}, \ldots, a_{s+1}$ in accordance with Lemma 1. From (1), (2) and (13),

where

$$
\frac{a_{s+1}-a_{1}}{n} S_{n}\left(x_{1}, \ldots, x_{n}\right)=\sum_{k=1}^{s} \phi_{d_{k}}\left(x_{a_{k}}, x_{a_{k}+1}, \ldots, x_{a_{k+1}+1}\right)
$$

$$
d_{k}=a_{k+1}-a_{k} \geqq 2 \text {, }
$$

from (10). From (31), (32) and Lemmas 2, 3 and 7,

$$
\frac{a_{s+1}-a_{1}}{n} S_{n}\left(x_{1}, \ldots, x_{n}\right) \geqq \sum_{\substack{k=1 \\ d_{k} \text { even }}}^{s} f_{d_{k}}\left(x_{a_{k}} / x_{a_{k+1}}\right)+\sum_{\substack{k=1 \\ d_{k} \text { odd }}}^{s} g_{d_{k}}\left(x_{a_{k}} / x_{a_{k+1}}\right),
$$

since (11) or (12) is satisfied. Hence, by (21) and (22),

$$
\begin{aligned}
& \frac{a_{s+1}-a_{1}}{n} S_{n}\left(x_{1}, \ldots, x_{n}\right) \geqq \sum_{\substack{k=1 \\
d_{k} \text { even }}}^{s} \frac{1}{2} d_{k} F_{d_{k}}\left(x_{a_{k}}^{2 / d_{k}} / x_{a_{k+1}}^{2 / d_{k}}\right) \\
&+\sum_{\substack{k=1 \\
d_{k} \text { odd }}}^{s} \frac{1}{2} d_{k} G_{d_{k}}\left(x_{a_{k}}^{2 / d_{k}} / x_{a_{k+1}}^{2 / d_{k}}\right) .
\end{aligned}
$$


A CYCLIC INEQUALITY AND AN EXTENSION OF IT 149 Suppose that $\alpha(1+\alpha) \leqq \frac{9}{4}$. Then, by (32), (33) and Lemmas 5, 6 and 9,

$$
\frac{a_{s+1}-a_{1}}{n} S_{n}\left(x_{1}, \ldots, x_{n}\right) \geqq \sum_{k=1}^{s} \frac{1}{2} d_{k} F_{2}\left(x_{a_{k}}^{2 / d_{k}} / x_{a_{k+1}}^{2 / d_{k}}\right) \geqq \frac{1}{2}\left(a_{s+1}-a_{1}\right) F_{2}(1),
$$

by the convexity property of $F_{2}(x)$, given in Lemma 4 , and the fact that, as a consequence of (2) and (9),

$$
\prod_{k=1}^{s}\left(x_{a_{k}} / x_{a_{k+1}}\right)=1
$$

Hence, by (25),

$$
\frac{1}{n} S_{n}\left(x_{1}, \ldots, x_{n}\right) \geqq \sqrt{\frac{\alpha}{1+\alpha}}-\frac{1}{2} \frac{\alpha}{1+\alpha} ;
$$

and so, from (3) and (4),

$$
\lambda(n) \geqq \lambda \geqq \sqrt{\frac{\alpha}{1+\alpha}}-\frac{1}{2} \frac{\alpha}{1+\alpha}
$$

when $\alpha(1+\alpha) \leqq \frac{9}{4}$. If $\alpha=1$ we get the inequality (6); and if $\alpha(1+\alpha)=\frac{9}{4}$, so that $\alpha=\frac{1}{2}(\sqrt{ } 10-1)$, we get the inequality (30). That this is the best inequality, obtainable from (35), for $\alpha(1+\alpha) \leqq \frac{9}{4}$ follows from Lemma 11 .

\section{Further Improvements of (6)}

We next consider $\alpha(\geqq 1)$ satisfying

$$
\alpha(1+\alpha) \leqq \frac{625}{256} \text {. }
$$

As in $\S 3$, we can obtain (33) in this case also. It is convenient to write

Then,

$$
\sum_{\substack{k=1 \\ d_{k} \neq 3}}^{s} d_{k}=p, \quad \sum_{\substack{k=1 \\ d_{k}=3}}^{s} d_{k}=q \text { and } p+q=N .
$$

$$
a_{s+1}-a_{1}=N \geqq p \geqq 0,
$$

from (32) and (37). It is also convenient to write, in conformity with (34),

$$
\prod_{\substack{k=1 \\ d_{k} \neq 3}}^{s} \frac{x_{a_{k}}}{x_{a_{k+1}}}=x \text { and } \prod_{\substack{k=1 \\ d_{k}=3}}^{s} \frac{x_{a_{k}}}{x_{a_{k+1}}}=\frac{1}{x} .
$$

Then, using (32), (37) to (39) and Lemmas 4 to 6 and 10, we get, from (33),

$$
\begin{aligned}
\frac{N}{n} S_{n}\left(x_{1}, \ldots, x_{n}\right) & \geqq \frac{1}{2} p F_{2}\left(x^{2 / p}\right)+\frac{1}{2} q G_{3}\left(x^{-2 / q}\right) \\
& \geqq-\frac{1}{2} p \frac{\alpha}{1+\alpha}+p\left(\frac{\alpha}{1+\alpha}\right)^{\frac{1}{2}} x^{1 / p}+\frac{2}{3} q\left(\frac{1}{1+\alpha}\right)^{\frac{1}{2}} x^{-3 / 2 q} \\
& \geqq-\frac{1}{2} p \frac{\alpha}{1+\alpha}+\left(p+\frac{2}{3} q\right)\left(\frac{1}{1+\alpha}\right)^{\frac{1}{2}} \alpha^{\frac{1}{2} p /\left(p+\frac{2}{2} q\right)}
\end{aligned}
$$


by the inequality of the means. Thus we get, using (37),

$$
\frac{1}{n} S_{n}\left(x_{1}, \ldots, x_{n}\right) \geqq H(\alpha, p)=-\frac{p \alpha}{2 N(1+\alpha)}+\frac{p+2 N}{3 N}\left(\frac{\alpha}{1+\alpha}\right)^{\frac{1}{2}} \alpha^{1-3 N /(p+2 N)} \text {. }
$$

Thus, from (3), (4), (38) and (40),

$$
\lambda(n) \geqq \lambda \geqq \max _{\alpha \leqq \alpha_{0}} \min _{0 \leqq p \leqq N} H(\alpha, p),
$$

where (36) is equivalent to $\alpha \leqq \alpha_{0}=\frac{\sqrt{689-8}}{16}=1 \cdot 14055$. Now

$$
\frac{\partial H}{\partial p}=-\frac{\alpha}{2 N(1+\alpha)}+\frac{\alpha}{3 N}\left(\frac{\alpha}{1+\alpha}\right)^{\frac{1}{2}}\left(1+\frac{3 N \log \alpha}{p+2 N}\right) \exp \frac{-3 N \log \alpha}{p+2 N}
$$

and, in virtue of (38),

From Lemma 12, it follows that

$$
\frac{3}{2} \log \alpha \geqq \frac{3 N \log \alpha}{p+2 N} \geqq \log \alpha .
$$

$$
\frac{1}{3 N}\left(\frac{1}{1+\alpha}\right)^{\frac{1}{2}}\left(1+\frac{3}{2} \log \alpha\right) \leqq \frac{\partial H}{\partial p}+\frac{\alpha}{2 N(1+\alpha)} \leqq \frac{1}{3 N}\left(\frac{\alpha}{1+\alpha}\right)^{\frac{1}{2}}(1+\log \alpha) .
$$

Hence, for all $p$ satisfying (38),

$$
\frac{\partial H}{\partial p} \leqq 0 \text { if }\left(\frac{\alpha}{1+\alpha}\right)^{\frac{1}{2}} \geqq \frac{2}{3}(1+\log \alpha) \text {, i.e. } \alpha \leqq \alpha_{1}=1 \cdot 08571
$$

and $\quad \frac{\partial H}{\partial p} \geqq 0$ if $\alpha\left(\frac{1}{1+\alpha}\right)^{\frac{1}{2}} \leqq \frac{2}{3}+\log \alpha, \quad$ i.e. $\alpha \geqq \alpha_{2}=1.09277$

(since $1 \leqq \alpha \leqq \alpha_{0}$ ). It is seen that $1<\alpha_{1}<\alpha_{2}<\alpha_{0}$.

If $\alpha_{1} \leqq \alpha \leqq \alpha_{2}$ then $\frac{\partial H}{\partial p}=0$ for some $p$ satisfying (38). For this $p$, from (40) and (42),

$$
\{\alpha(1+\alpha)\}^{\frac{1}{2}}=\frac{3}{2}\left(1+\frac{3 N \log \alpha}{p+2 N}\right)^{-1} \exp \frac{3 N \log \alpha}{p+2 N}
$$

and

$$
H(\alpha, p)=\frac{1}{2} \frac{\alpha}{1+\alpha}\left\{2-3 \log \alpha\left(1+\frac{3 N \log \alpha}{p+2 N}\right)^{-1}\right\}
$$

If $\alpha \leqq \alpha_{1}, \frac{\partial H}{\partial p} \leqq 0$ for all $p$. Thus, from (40),

$$
\min _{0 \leqq p \leqq N} H(\alpha, p)=H(\alpha, N)=\int \frac{\alpha}{1+\alpha}-\frac{1}{2} \frac{\alpha}{1+\alpha} .
$$

Hence we have (35) and thus, from Lemma 11, the best inequality obtainable from (35) is when $\alpha=\alpha_{1}$. This is

$$
\lambda(n) \geqq \lambda \geqq 0 \cdot 461216 \text {. }
$$




\section{A CYCLIC INEQUALITY AND AN EXTENSION OF IT 151}

If $\alpha \geqq \alpha_{2}, \frac{\partial H}{\partial p} \geqq 0$ for all $p$. Thus, from (40),

and we have

$$
\min _{0 \leqq p \leqq N} H(\alpha, p)=H(\alpha, 0)=\frac{2}{3} \sqrt{\frac{1}{1+\alpha}} ;
$$

$$
\lambda(n) \geqq \lambda \geqq \frac{2}{3} \sqrt{\frac{1}{1+\alpha}}
$$

which is best when $\alpha=\alpha_{2}$. The inequality then is

$$
\lambda(n) \geqq \lambda \geqq 0.460838 \text {, }
$$

which is not so good as (45).

If $\alpha_{1} \leqq \alpha \leqq \alpha_{2}$, from (43) and (44) we find (by computation) that, for $\alpha$ and $p$ satisfying (43), $H(\alpha, p)$ has its maximum value when $\alpha=1.0868$ and $p=0.7214 N$. This maximum value is 0.461238 . Thus (41) is equivalent to (7) which, we note, is only a slight improvement of (45), which itself is better than (6).

\section{Proof of (8)}

From (1) and (3), we easily get (8) if we let $n$ be 24 and $x_{1}, \ldots, x_{24}$ be 0,15 , $0,17,0,19,0,21,2,22,5,21,7,18,7,16,6,14,5,13,3,13,1,14$ respectively, in (1), and use considerations of continuity.

\section{Addendum to (1)}

Near the end of (1) we proved an inequality equivalent to

$$
\Sigma_{n}\left(x_{1}, \ldots, x_{n}\right)=\frac{4}{n} \sum_{r=1}^{n} \frac{x_{r}}{3 x_{r+1}+x_{r+2}+\left|x_{r+1}-x_{r+2}\right|} \geqq 2^{\left[\frac{1}{2} n\right] / n}-\frac{\left[\frac{1}{2} n\right]}{n} \ldots
$$

if (2) is satisfied. We can now prove more, namely, that

if (2) is satisfied.

$$
\inf _{x_{1}, \ldots x_{n}} \Sigma_{n}\left(x_{1}, \ldots, x_{n}\right)=2^{\left[\frac{1}{2} n\right] / n}-\frac{\left[\frac{1}{2} n\right]}{n}
$$

Proof. For even $n$, (47) follows from (46) since we have equality in (46) if

$$
x_{1}=x_{3}=\ldots=x_{n-1} \text { and } x_{2}=x_{4}=\ldots=x_{n}=(\sqrt{ } 2-1) x_{1} \text {, }
$$

when

$$
\Sigma_{n}=\frac{x_{1}}{x_{2}+x_{3}}+\frac{x_{2}}{2 x_{3}}=\sqrt{ } 2-\frac{1}{2} \text {. }
$$

For $n=1$, (47) is trivially true. For odd $n>1$, (47) follows from (46) since we have equality in (46) if

$$
x_{1}: x_{2}: x_{3}=x_{3}: x_{4}: x_{5}=\ldots=x_{n-2}: x_{n-1}: x_{n}=2^{-1 / n}: 2^{(n-1) / 2 n}-1: 1 \text {, }
$$

when

$$
\Sigma_{n}=\frac{n-1}{n}\left(\frac{x_{1}}{x_{2}+x_{3}}+\frac{x_{2}}{2 x_{3}}\right)+\frac{x_{n}}{n x_{1}}=2^{(n-1) / 2 n}-\frac{n-1}{2 n} \text {. }
$$




\section{REFERENCES}

(1) P. H. Diananda, A cyclic inequality and an extension of it. I, Proc. Edin. Math. Soc., 13 (1962), 79-84.

(2) P. H. Diananda, On a cyclic sum, Proc. Glasgow Math. Assoc. (to appear).

(3) G. H. Hardy, J. E. Littlewood and G. Pólya, Inequalities (Cambridge, 1934).

(4) R. A. Rankin, An inequality, Math. Gaz., 42 (1958), 39-40.

(5) R. A. Rankin, A cyclic inequality, Proc. Edin. Math. Soc., 12 (1961), 139-147.

(6) A. Zulauf, On a conjecture of L. J. Mordell II, Math. Gaz. 43 (1959), 182-184.

Department of Mathematics

THE UNIVERSITY

SINGAPORE, 10 